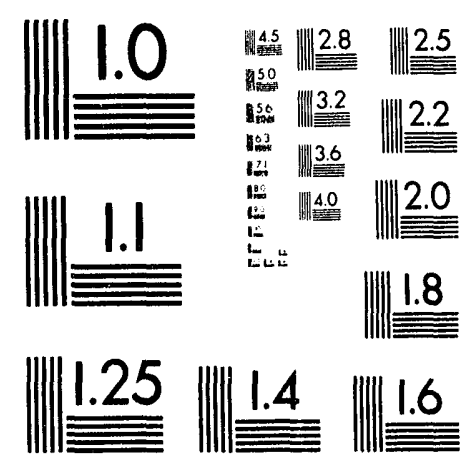



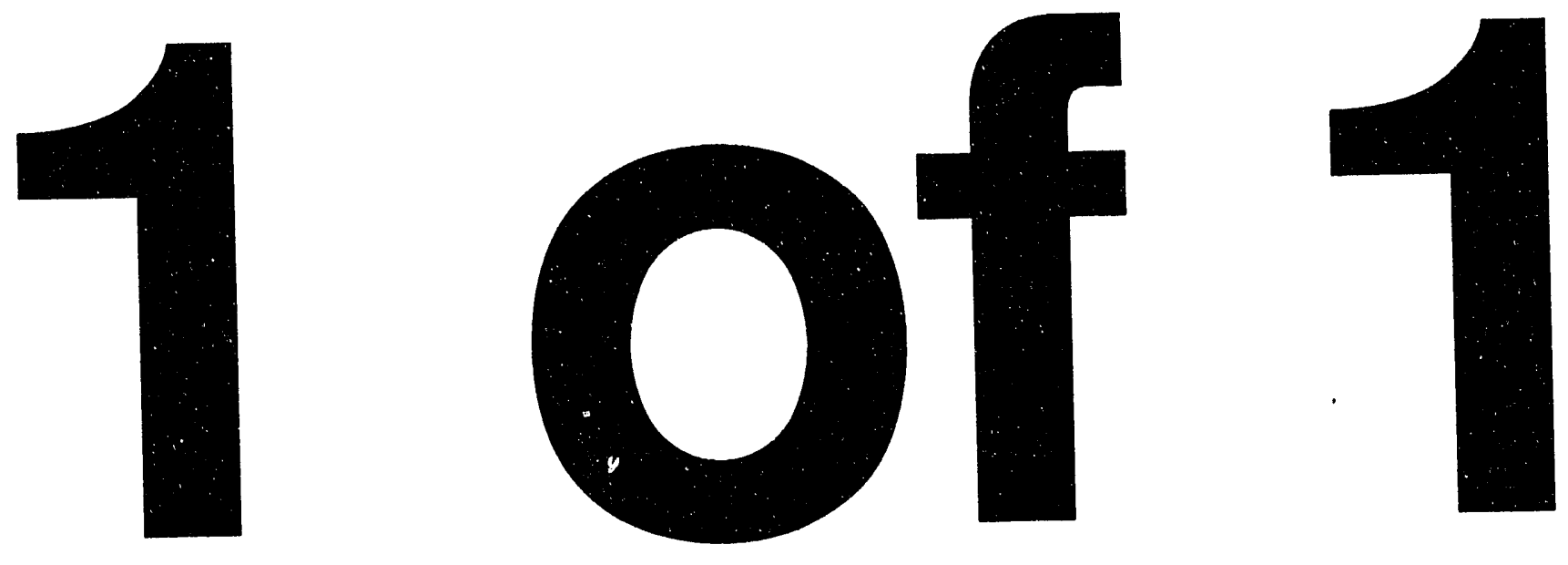


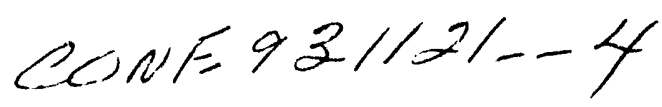

SAND92-2487C

To be presented at the American Society of Mechanical Engineers Winter Annual Meeting Symposium on Plastics and Plastic Composites, New Orleans, Louisiana, November 1993.

\title{
A CONSTITUTIVE THEORY FOR RIGID POLYURETHANE FOAM
}

\author{
M.K. Neilsen \\ Engineering Mechanics and Material Modeling Department \\ Sandia National Laboratories \\ Albuquerque, New Mexico 87185 \\ Prof. R.D. Krieg \\ Department of Engineering Science and Mechanics \\ University of Tennessee \\ Knoxville, TN 37996 \\ Prof. H.L. Schreyer \\ Department of Mechanical Engineering \\ University of New Mexico \\ Albuquerque, NM 87131
}

\begin{abstract}
Rigid, closed-cell, polyurethane foam consists of interconnected polyurethane plates which form cells. When this foam is compressed, it exhibits an initial elastic regime which is followed by a plateau regime in which the load required to compress the foam remains nearly constant. In the plateau regime, cell walls are damaged and large permanent volume changes are generated. As additional load is applied, cell walls are compressed against neighboring cell walls, and the stiffness of the foam increases and approaches a value equal to that of solid polyurethane. When the foam is loaded in tension, the cell walls are damaged and the foam fractures. A constitutive theory for rigid polyurethane foam has been developed. This theory is based on a decomposition of the foam into two parts: a skeleton and a nonlinear elastic continuum in parallel. The skeleton accounts for the foam behavior in the elastic and plateau regimes and is described using a coupled plasticity with continuum damage theory. The nonlinear elastic continuum accounts for the lock-up of the foam due to internal gas pressure and cell wall interactions. This new constitutive theory has been implemented in both static and dynamic finite element codes. Numerical simulations performed using the new constitutive theory are presented.
\end{abstract}




\section{DISCLAIMER}

This report was prepared as an account of work sponsored by an agency of the United States Government. Neither the United States Government nor any agency thereof, nor any of their employees, makes any warranty, express or implied, or assumes any legal liability or responsibility for the accuracy, completeness, or usefulness of any information, apparatus, product, or process disclosed, or represents that its use would not infringe privately owned rights. Reference herein to any specific commercial product, process, or service by trade name, trademark, manufacturer, or otherwise does not necessarily constitute or imply its endorsement, recommendation, or favoring by the United States Governmont or any agency thereof. The views and opinions of authors expressed herein do not necessarily state or reflect those of the United States Government or any agency thereof. 


\section{EXPERIMENTAL OBSERVATIONS}

Rigid, closed-cell, polyurethane foam consists of interconnected polyurethane plates. This material is manufactured by having a gas expand inside liquid polyurethane that later cools and solidifies in a foamed state. Shaw and Sata (1966), Patel and Finnie (1969), Zaslawsky (1973), Triantafillou et al. (1989), and Donald and Maji (1992) have experimentally measured the behavior of polyurethane foam samples subjected to various load paths. Patel and Finnie's (1969) work indicates that the initial failure surface for this material subjected to plane stress states has the rectangular shape shown in Figure 1. When the foam is compressed beyond its elastic limit, it is both plastically deformed and damaged. When the foam is loaded in tension beyond its elastic limit, cell walls are damaged and the foam quickly fractures. The experimental work of Shaw and Sata (1966), Zaslawsky (1973), and Donald and Maji (1992) indicates that the initial yield surface for rigid polyurethane foam can be described as six intersecting planes whose normals are the principal stress axes. Experiments performed by Trantafillou et al. (1989) are generally in agreement with those of other researchers; however, their experiments show that the application of radial compressive loads to an axisymmetric sample subjected to axial tension will decrease the axial tensile strength of the sample.

A load-displacement curve for $80 \mathrm{~kg} / \mathrm{m}^{3}$ polyurethane foam subjected to either uniaxial compression or uniaxial tension is shown in Figure 2. For uniaxial compression, the initial elastic regime is followed by a plateau regime in which the load required to crush the material remains nearly constant. In this regime, some cell walls are compressed until they buckle while other cell walls are stretched until they crack. When additional load is applied, cell walls are compressed against neighboring cell walls and the stiffness of the foam approaches a value equal to the stiffness of solid polyurethane. Other experiments performed by Donald and Maji (1992) reveal that the foam stiffness decreases a small amount when the material is compressed into the plateau regime but that the foam stiffness increases when it is compressed further into the lock-up regime. The decrease and subsequent increase in foam stiffness is caused by two distinct deformation mechanisms. The decrease in stiffness occurs due to the cracking and reduced stiffness of the cell walls. The increase in stiffness is due to the interaction between neighboring cell walls as they are compressed together. When the foam is loaded in tension, the cell walls elastically deform and then fracture. The axial deformations localize into a very thin zone which has a normal parallel to the loading axis when the cell walls fracture.

\section{EXISTING CONSTITUTIVE THEORIES}

Several researchers (Gibson and Ashby, 1988; Warren and Kraynik, 1987) have studied the linear elastic behavior of various cellular solids. In this work, equations are developed that relate the elastic constants to parameters that define the geometry of a single cell (i.e., the thickness of the cell wall and length of a typical cell wall section). Equations that define the critical load beyond which the behavior is no 
longer linear elastic are also developed by these researchers. These equations are then used to describe the linear elastic behavior of a group of cells by assuming that all cells have the same initial geometry and deform in the same manner. This work was recently extended (Warren, Kraynik and Stone, 1989) to capture the nonlinear elastic behavior of two-dimensional open cell foams.

Rigid foam is often used in packaging or crash padding to protect some object during an accidental impact event. During a typical accident, the foam is expected to absorb a significant amount of energy by undergoing large inelastic deformations. Several researchers (Rusch, 1969 and 1970; Ramon, Mizrahi and Miltz, 1990; Sherwood and Frost, 1992) have used a phenomenological approach to develop expressions which describe the inelastic response of foams to uniaxial compression. Most packages and crash pads are currently designed using these expressions. The uniaxial load-displacement curves are used to compute the amount of energy absorbed by the foam for a given uniaxial impact event. This method is accurate for describing the response of impact limiting materials subjected to uniaxial loads; however, this simple uniaxial approach cannot be applied to situations involving multiaxial loading. A general continuum representation is needed if multiaxial loading is involved. The uniaxial descriptions are adequate for only simple package geometries and loadings.

A few researchers are currently working on the development of continuum constitutive theories that describe both the elastic and inelastic responses of cellular materials to complex load paths. A variety of constitutive theories have been proposed to describe this complex material. In 1972, Krieg developed a plasticity theory for soils and crushable foams. This plasticity theory has a yield surface which is a paraboloid of revolution about the hydrostat with a planar cap on the normally open end. Neilsen, Morgan and Krieg (1987) developed a plasticity theory for rigid, polyurethane foams which has six intersecting planar yield surfaces which form a cubic shape in principal stress space. Triantafillou and Gibson (1991) proposed a failure surface for cellular materials which is an ellipsoid elongated along the hydrostat that is truncated in the tensile and compressive octants by box-like surfaces which account for brittle fracture and cell wall buckling. Based on Trantafillou et al.'s (1989) experimental work, Theocaris (1992) proposed an initial failure surface for closed-cell polyurethane foams which is described as an elliptic paraboloid in principal stress space that is truncated by ellipsoidal failure surfaces which account for brittle fracture and cell wall buckling. The plasticity model for rigid polyurethane foam, developed by Neilsen, Morgan and Krieg (1987), is used as the basis for the coupled plasticity with continuum damage model for polyurethane foam presented in this paper.

\section{DESCRIPTION OF THE FOAM AS A MIXTURE}

The first step in the development of a constitutive theory for polyurethane foams was an examination of the individual components of the foam structure. Rigid polyurethane foams consist of connected, closed polyurethane cells with air inside the cells. Therefore, each foam consists of the following structural components: (1) the polyurethane structure or skeleton, (2) the polyurethane material that makes up 
the cell walls, and (3) the air inside the cells. In applications where the air cannot escape from the skeleton during loading, the air can carry a substantial part of the load. For example, in hydrostatic and triaxial compression tests which were performed by Donald and Maji (1992), the samples were jacketed, and the air could not escape. Thus, a model which considers the contribution of the air to the overall structural response of the foams is needed to capture the foam behavior exhibited in these tests.

Total foam response can be decomposed intu the response of a skeleton and the response of a diffuse continuum of air and polyurethane particles as shown in Figure 3. We assume that the diffuse continuum of air and polyurethane particles does not resist any shear deformation; thus, the air/polyurethane contribution is completely volumetric. For convenience, the skeleton is assumed to occupy the same space as the foam. This implies that the skeleton strain tensor is equal to the foam strain tensor. Also, the foam stress tensor, $\boldsymbol{\sigma}^{\text {foam }}$, is given by the following equation

$$
\boldsymbol{\sigma}^{\text {foam }}=\boldsymbol{\sigma}^{s k}+p \mathbf{i}
$$

where $\mathbf{i}$ is the second order identity tensor, $\boldsymbol{\sigma}^{s k}$ is the skeleton stress tensor, and $p \mathbf{i}$ represents the diffuse continuum contribution. To better understand this equation, consider a hydrostatic compression test in which the foam sample is jacketed and the air is not allowed to escape. If the foam consisted of loosely connected particles of polyurethane, then the skeleton contribution to the foam response would be negligible and the external pressure applied to the foam would equal the pressure in the diffuse continuum of air and polyurethane particles. In other words, the foam stress would equal the diffuse continuum stress. This foam would not be able to resist any deviatoric loading. In most foams, however, the skeleton is structured so that it can carry load, and the contribution of the skeleton must be added to the diffuse continuum contribution to determine how much load the foam can carry. In the next section, an expression for the diffuse continuum contribution is derived.

\section{DIFFUSE CONTINUUM RESPONSE}

An expression for the pressure in the diffuse continuum, $p$, was obtained by assuming that: 1. the air is in series with the polyurethane particles, 2. the air is a perfect gas, and 3. the foam compression is an isothermal process. The isothermal assumption is made because the air is in intimate contact with the polyurethane that makes up the cell walls and the thermal mass of the polyurethane is significantly larger than the thermal mass of the air. These equations indicate that the perfect gas equation of state can be used for the air and that

$$
p^{a i r} V^{a i r}=p_{o}^{a i r} V_{o}^{a i r}
$$

where $p^{a i r}$ is the current absolute air pressure, $V^{\text {air }}$ the current air volume, $p_{o}^{\text {air }}$ the original air pressure, and $V_{o}^{a i r}$ the original air volume. The original air volume is related to the original foam volume, $V_{o}$, by

$$
V_{o}^{a i r}=V_{o}(1-\Phi)
$$


where $\Phi$ is the volume fraction of solid material in the undeformed sample. Prior to loading, we assume that the internal air pressure, $p_{0}^{a i r}$, is in equilibrium with the environment, i.e., $p_{0}^{a i r} \approx 101 \mathrm{kPa}$. The change in internal air pressure due to the loading $p=p^{a i r}-p_{o}^{a i r}$ is related to the change in air volume as follows:

$$
\Delta V^{a i r}=\frac{-p V_{o}(1-\Phi)}{p_{o}^{a i r}+p}
$$

The volume of the polyurethane which makes up the cell walls will change according to the bulk modulus of the polyurethane, $K^{\text {poly }}$, as follows:

$$
\Delta V^{\text {poly }}=\frac{-p V_{o} \Phi}{K^{\text {poly }}}
$$

The engineering volume strain, $e_{v o l}$, is given by

$$
e_{\text {vol }}=\frac{\Delta V^{a i r}+\Delta V^{\text {poly }}}{V_{o}}
$$

Finally, by combining the above equations, the following quadratic equation is obtained

$$
\Phi p^{2}+\left[p_{o}^{a i r} \Phi+K^{\text {poly }}\left(1-\Phi+e_{v o l}\right)\right] p+e_{v o l} K^{\text {poly }} p_{o}^{a i r}=0
$$

This equation can be easily solved to obtain an expression for the pressure in the diffuse continuum, $p$. Only one root is shown to be physically realistic. A plot of air pressure as a function of volume strain using Equation 7 is shown in Figure 4. If the air is allowed to escape then the polyurethane particle response is given by Equation 7 with the initial air pressure, $p_{0}^{a i r}$ set equal to zero. With $p_{0}^{\text {air }}$ equal to zero, Equation 7 has two roots

$$
p=0 . \quad \text { or } \quad p=-\frac{K^{\text {poly }}}{\Phi}\left(1-\Phi+e_{\text {vol }}\right)
$$

These equations describe two intersecting lines as shown in Figure 5. When the air is allowed to escape, these equations indicate that the diffuse continuum does not contribute to the foam response until the engineering volume strain, $e_{v o l}$, is larger in magnitude than $1-\Phi$.

\section{COUPLED THEORY FOR THE SKELETON}

A combination of plasticity and continuum damage theories are used to describe the inelastic deformation of the skeleton. Plasticity is used to describe the permanent deformation of the cell walls when the skeleton is compressed. Continuum damage is used to capture the change in elastic response due to cell wall damage. A number of finite element codes have been developed at Sandia National Laboratories to simulate the finite deformations of nonlinear materials subjected to both static and dynamic loads (Stone, 1992; Taylor and Flanagan, 1987). In these codes, the constitutive theories are expressed in terms of the unrotated Cauchy stress, $\boldsymbol{\sigma}$, and the deformation rate, $\mathbf{d}$, in the unrotated configuration (Taylor and Flanagan, 1987). 
Plasticity theories are characterized by their yield function, $\Psi$, and their evolution equation for plastic deformation. Likewise, continuum damage theories can be characterized by a damage function and an evolution equation for either the fourth-order stiffness tensor, $\mathbf{E}$, or the fourth-order compliance tensor, $\mathbf{C}$, for the material. The compliance is simply equal to the inverse of the stiffness. The yield or damage function defines a surface in stress space which bounds stress states for which the response of the material is elastic. The evolution equations define the nature of the plastic deformation or damage.

The experimental work of Shaw and Sata (1966), Patel and Finnie (1969), Zaslawsky (1973), and Donald and Maji (1992) indicates that the initial yield (damage) surface for rigid polyurethane foam can be described using a principal stress criterion; thus, we adopt the following yield (damage) function for the skeleton

$$
\Psi^{i}=\frac{1}{2}\left(\boldsymbol{\sigma}: \mathbf{P}^{\mathrm{i}}: \boldsymbol{\sigma}\right)^{\frac{1}{2}}-\left(A+B e_{\text {vol }}\right) \quad i=1,2,3
$$

where $A$ and $B$ are material parameters which depend on the sign of the principal stress and $\mathbf{P}^{i}$ is the fourth order principal projection operator given by

$$
\mathbf{P}^{i}=\mathbf{n}^{i} \otimes \mathbf{n}^{i} \otimes \mathbf{n}^{i} \otimes \mathbf{n}^{i} \quad i=1,2,3
$$

where $\mathbf{n}^{i}$ is a vector oriented in a principal stress direction. The yield (damage) functions, $\Psi^{i}=0$, actually represent 3 pairs of planar yield surfaces with normals given by $\mathrm{n}^{i}$. We assume that the yield and damage functions are identical for the skeleton.

The evolution equations for plastic strain and material compliance are given by

$$
\mathbf{d}^{\text {plastic }}=\dot{\alpha}^{1} \mathbf{P}^{1}: \boldsymbol{\sigma}+\dot{\alpha}^{2} \mathbf{P}^{2}: \boldsymbol{\sigma}+\dot{\alpha}^{3} \mathbf{P}^{3}: \boldsymbol{\sigma} \quad \dot{\mathbf{C}}^{\text {damage }}=\dot{\beta} \mathbf{C}^{\text {elastic }}
$$

where $\mathbf{C}^{\text {elastic }}$ is the original undamaged elastic compliance tensor and $\mathbf{C}^{\text {damage }}$ is the additional material compliance due to damage. The evolution equation for plastic strain indicates that a permanent deformation increment may be associated with each principal stress direction depending on the magnitude of the principal stress. The damage evolution equation indicates that the material damage is given by a single scalar $\beta$ such that the current compliance, $\mathrm{C}$, of the material is as follows:

$$
\mathbf{C}=\mathbf{C}^{\text {elastic }}+\mathbf{C}^{\text {damage }}=(1+\beta) \mathbf{C}^{\text {elastic }}
$$

The total rate of deformation or stretching tensor is additively decomposed into elastic, damaging, and plastic parts as follows:

$$
\mathbf{d}=\mathbf{d}^{\text {elastic }}+\mathbf{d}^{\text {damage }}+\mathbf{d}^{\text {plastic }}
$$

where

$$
\begin{gathered}
\mathbf{d}^{\text {elastic }}=\mathrm{C}^{\text {elastic }}: \dot{\boldsymbol{\sigma}} \\
\mathrm{d}^{\text {damage }}=\mathrm{C}^{\text {damage }}: \dot{\boldsymbol{\sigma}}+\dot{\mathrm{C}}^{\text {damage }}: \boldsymbol{\sigma}
\end{gathered}
$$


The above equations can be combined to obtain the following expression for the stress rate associated with a given stretching rate

$$
\dot{\boldsymbol{\sigma}}=\mathbf{E}^{\text {elastic }}: \mathbf{d}^{\text {elastic }}=\mathbf{E}^{\text {elastic }}:\left(\mathbf{d}-\mathbf{d}^{\text {damage }}-\mathbf{d}^{\text {plastic }}\right)
$$

where the fourth-order undamaged elastic stiffness tensor, $\mathbf{E}^{\text {elastic }}$, is the inverse of the undamaged elastic compliance tensor $\mathbf{C}^{\text {elastic }}$. In the elastic regime, inside the yield (damage) surface, $\dot{\alpha}^{i}$ and $\dot{\beta}$ are equal to zero, and the constitutive relation, Equation 16 , reduces to

$$
\dot{\boldsymbol{\sigma}}=\frac{1}{(1+\beta)} \mathbf{E}^{\text {elastic }}: \mathbf{d}=\mathbf{E}: \mathbf{d}
$$

where $\mathbf{E}$ is the current stiffness tensor which includes the effects of prior damage. The coupled theory for the skeleton assumes that the elastic response is linear and isotropic; thus, $\mathbf{E}^{\text {elastic }}$ and $\mathbf{C}^{\text {elastic }}$ are as follows:

$$
\mathbf{E}^{\text {elastic }}=3 K \mathbf{P}^{s p}+2 G \mathbf{P}^{d} \quad \mathbf{C}^{\text {elastic }}=\frac{1}{3 K} \mathbf{P}^{s p}+\frac{1}{2 G} \mathbf{P}^{d}
$$

where $K$ is the bulk modulus and $G$ is the shear modulus. The fourth order spherical projection operator, $\mathbf{P}^{s p}$, and the deviatoric projection operator, $\mathbf{P}^{d}$, are given by

$$
\mathbf{P}^{s t}=\frac{1}{3} \mathbf{i} \otimes \mathbf{i} \quad \mathbf{P}^{d}=\mathbf{I}-\mathbf{P}^{s p}
$$

where $\mathbf{I}$ is the symmetric fourth order identity tensor, and $\mathbf{i}$ is the second order identity. Since Poisson's ratio, $v$, for the skeleton is equal to zero, $3 K=2 G=E$ and $\mathbf{E}^{\text {elastic }}$ and $\mathbf{C}^{\text {elastic }}$ are as follows:

$$
\mathbf{E}^{\text {elastic }}=E \mathbf{I} \quad \mathbf{C}^{\text {elastic }}=\frac{1}{E} \mathbf{I}
$$

where $E$ is Young's modulus for the skeleton.

To complete this model we need an additional equation that prescribes the relative amounts of damage and permanent strain generated during an inelastic step. The additional equation is given by

$$
\mathbf{d}^{\text {damage }}=M\left(\mathbf{d}^{\text {damage }}+\mathbf{d}^{\text {plastic }}\right)
$$

In this equation, we allow the magnitude of the state variable $M$ to depend on the sign of the principal stress and to vary between zero and one. When the principal skeleton stresses are compressive, the inelastic behavior of the skeleton is dominated by vlasticity so for compressive principal stresses $M$ is given a small value. When the principal skeleton stresses are tensile, the skeleton fractures and exhibits only damage; thus, for tensile principal stresses $M$ is given a value of one. 


\section{ANALYSIS OF MATERIAL CHARACTERIZATION TESTS}

A series of experiments were recently performed by Donald and Maji (1992) to characterize the behavior of rigid polyurethane foams and aluminum honeycombs. In this section, several finite element analyses of the material characterization tests are performed to determine if the constitutive theory developed in this paper will predict the foam behaviors which are observed experimentally.

The analyses were performed using the finite element models of axisymmetric material specimens shown in Figure 6 and the code SANTOS (Stone, 1992). The models were subjected to uniaxial compression, hydrostatic compression and uniaxial tension. The predicted response of the foam from the finite element analyses was compared with the experimental observations. Properties that are appropriate for an $80 \mathrm{~kg} / \mathrm{m}^{3}$ foam were used in this investigation (Table 1).

In the initial analyses, the models were subjected to uniaxial compression. A plot of the undeformed and deformed 200 element mesh is shown in Figure 7. The deformed shapes show that the model predicts only axial deformation when the material is compressed in the plateau regime and a combination of axial and radial deformation when the material is loaded into the lock-up regime. This deformation behavior is observed experimentally. Mesh refinement had no significant effect on the predicted compressive response of the material. A plot of the applied load versus end displacement predicted by the finite element analyses is compared with the experimental result in Figure 8. This plot shows that the response predicted by the finite element analyses is qualitatively accurate; however, the amount of axial deformation required to lock-up the foam is smaller than the axial deformation at lock-up predicted by the analyses. One possible explanation for this discrepancy is that the expression for the diffuse continuum contribution is based on the assumption that the polyurethane particles which make up the cell walls are in series with the air. Thus, lock-up is predicted when the engineering volume strain, $e_{v o l}$, obtains a magnitude of $1-\Phi$. The polyurethane particles which make up the cell walls actually begin to contact each other and foam lock-up begins prior to this point. A more accurate description of the diffuse continuum contribution should be developed from a careful study of the foam microstructure at lock-up.

In the next analyses, the models were subjected to uniaxial tension. A plot of the deformed meshes (Figure 9) show that the axial deformations localize into a narrow zone which has a normal parallel to the loading axis. This is exactly the type of failure which was observed in the uniaxial tension experiments performed by Donald

Table 1. Material Parameters for a $80 \mathrm{~kg} / \mathrm{m}^{3}$ Polyurethane Foam

\begin{tabular}{|c|c|c|c|c|c|c|c|c|}
\hline $\begin{array}{c}\text { Elastic } \\
\text { Modulus } \\
(\mathrm{MPa})\end{array}$ & $\begin{array}{c}\text { Poisson's } \\
\text { Ratio }\end{array}$ & $\begin{array}{c}\mathrm{A} \\
\text { Comp. } \\
(\mathrm{kPa})\end{array}$ & $\begin{array}{c}\mathrm{B} \\
\text { Comp. } \\
(\mathrm{kPa})\end{array}$ & $\begin{array}{c}\mathrm{M} \\
\text { Comp. }\end{array}$ & $\begin{array}{c}\mathrm{A} \\
\text { Tension } \\
(\mathrm{kPa})\end{array}$ & $\begin{array}{c}\mathrm{B} \\
\text { Tension } \\
(\mathrm{psi})\end{array}$ & $\begin{array}{c}\mathrm{M} \\
\text { Tension }\end{array}$ & $\Phi$ \\
\hline 20.7 & 0.0 & 758 & 0 & 0.1 & 758 & -690 & 1.0 & 0.09 \\
\hline
\end{tabular}


and Maji (1992). A plot of the applied load versus end displacement predicted by the finite element analyses is compared with the experimental result in Figure $10 . \Lambda \mathrm{s}$ expected, the predicted load-displacement is clearly mesh dependent in the softening regime when localization occurs. One way to eliminate this mesh dependance would be to use a non-local constitutive theory for the skeleton (Schreyer and Chen, 1986).

In the final analyses, the models were subjected to hydrostatic compression. Plots of the deformed meshes are shown in Figure 11. The size of the original undeformed sample is indicated by the dashed lines in Figure 11. These analyses predict the generation of rather unusual deformed shapes during the hydrostatic compression tests which is consistent with experimental observations. A plot of applied pressure versus engineering volume strain predicted by the finite element analyses is compared with the experimental result in Figure 12. This plot shows that the response predicted by the finite element analyses matches the experimental result reasonably well. During the experiment, the foam was not compressed to lock-up due to pressure chamber limitations. Also, even though the deformed shapes were rather mesh dependent the plots of applied pressure vs. volume strain were not mesh dependent.

\section{CONCLUSIONS}

The behavior of rigid, closed-cell, polyurethane foam has been experimentally investigated by a number of researchers. Results from these experiments reveal that when these foams are compressed, they exhibit an initial elastic regime which is followed by a plateau regime in which the load required to compress the foam remains nearly constant. In the plateau regime, cell walls are damaged, and large permanent volume changes are generated. As additional load is applied, cell walls are compressed against neighboring cell walls, and the stiffness of the foam increases and approaches a value equal to that of solid polyurethane. When the foam is loaded in tension, the cell walls are damaged, and the foam fractures. To determine the shape of the yield (damage) surface, experimentalists must subject the foam to a variety of complex load paths and measure the foam response. Some disagreement currently exists as to what the initial yield (damage) surface for rigid, polyurethane foam looks like. This is probably due to the fact that it is very difficult to experimentally subject a foam sample to a truly uniform complex stress state because foam samples exhibit large deformations prior to the initiation of plastic deformation or damage. We may need to resort to a combination of analyses and experiments to fully understand the behavior of foam and the stress states that are actually generated in even the simplest experiments.

The constitutive theory developed in this paper describes both the elastic and inelastic responses of rigid polyurethane foams subjected to complex load paths. This theory is based on a decomposition of the foam into two parts: a skeleton and a nonlinear elastic continuum in parallel. The skeleton accounts for the foam behavior in the elastic and plateau regimes and is described using a coupled plasticity with continuum damage theory. The nonlinear elastic continuum accounts for the lock-up of the foam dun to internal gas pressure and cell wall interactions. This new constitutive theory qualitatively captures rigid, polyurethane foam behavior. A number of 
material characterization tests were analyzed using the new constitutive theory and the finite element code SANTOS (Stone, 1992). Results from these simulations indicate that our diffuse continuum description is not adequate and must be modified to account for all of the mechanisms which contribute to foam lock-up. A careful study of the foam microstructure at lock-up would enhance our understanding of the mechanisms responsible for lock-up. Dynamic experiments on rigid polyurethane foams indicate that the compressive strength of the material increases with strain rate. This behavior could be captured by using a rate-dependent constitutive theory to describe the behavior of the skeleton. Also, the lock-up behavior of the foam may or may not be affected by strain rate. Once rate effects are incorporated into this constitutive theory, it can be used in the numerical simulation of complex package impact events.

\section{ACKNOWLEDGMENT}

This work was supported by the United States Department of Energy under Contract DE-AC04-76DP00789 with Sandia National Laboratories.

\section{REFERENCES}

Donald, S. and Maji, A.K., 1992,Static Testing of Aluminum Honeycomb and Polyurethane Foams, contractors report to Sandia National Laboratories, Albuquerque, NM.

Gibson, L.J. and Ashby, M.F., 1988,Cellular Solids - Structure and Properties, Pergamon Press, New York.

Krieg, R.D., 1972,A Simple Constitutive Description for Soils and Crushable Foams, SC-DR-72-0883, Sandia National Laboratories, Albuquerque, NM.

Neilsen, M.K., Morgan, H.S., and Krieg, R.D., 1987, A Phenomenological Constitutive. Model for Low Density Polyurethane Foams, SAND86-2927, Sandia National Laboratories, Albuquerque, NM.

Patel, N.R. and Finnie, I., 1969, The Deformation and Fracture of Rigid Cellular Plastics Under Multiaxial S'tress, Report UCRL-13420, Lawrence Livermore Laboratory, Livermore, CA.

Ramon, O., Mizrahi, S., and Miltz, J., 1990, Mechanical Properties and Behavior of Open Cell Foams Used as Cushioning Materials, Polym. Eng. Sci., Vol. 30, pp. $197-201$.

Rusch, K.C., 1969, Load-Compression Behavior of Flexible Foams, J. Appl. Polym. Sci., Vol. 13, pp. 2297-2311.

Rusch, K.C., 1970, Energy Absorbing Characteristics of Foamed Polymers, J. Appl. Polym. Sci., Vol. 14, pp. 1433-1447. 
Schreyer, H.L. and Chen, Z., 1986, One-Dimensional Softening with Localization, J. Appl. Mech., Vol. 53, pp. 791-797.

Shaw, M.C. and Sata, T., 1966, The Plastic Behavior of Cellular Materials, Int. J. Mech. Sci., Vol. 8, pp. 469-478.

Sherwood, J.A. and Frost, C.C., 1992, Constitutive Modeling and Simulation of Energy Absorbing Polyurethane Foam Under Impact Loading, Polym. Eng. Sci., Vol. 32, pp. 1138-1146.

Stone, C. M., 1992,SANTOS - A Two-Dimensional Finite Element Program for the Quasistatic, Large Deformation, Inelastic Response of Solids, SAND90-0543, Sandia National Laboratories, Albuquerque, NM.

Taylor, L. M. and Flanagan, D. P., 1987,PRONTO2D - A Two-Dimensional Transient Solid Dynamics Program, SAND86-0594, Sandia National Laboratories, Albuquerque, NM.

Theocaris, P.S., 1992, Failure Modes of Closed-Cell Polyurethane Foams, Intl. J. Fract., Vol. 56, pp. 353-375.

Triantafillou, T.C., and Gibson, L.J., 1991,Multiaxial Failure Criteria for Cellular Materials, Mat. Res. Soc. Symp. Proc., Vol. 207, pp. 9-14.

Triantafillou, T.C., Zhang, Z., Shercliff, T.L., Gibson, L.J., and Ashby, M.F., 1989,Failure Su.faces for Cellular Materials Under Multiaxial Loads - Comparison of Models with Experiment, Int. J. Mech. Sci., Vol. 31, pp. 665-678.

Warren, W.E., Kraynik, A.M., 1987,Foam Mechanics: The Linear Elastic Response of Two-Dimensional Spatially Periodic Cellular Materials, Mech. Mater., Vol 6, pp. 27-37.

Warren, W.E., Kraynik, A.M., and Stone, C.M., 1989, A Constitutive Model for TwoDimensional Nonlinear Elastic Foams, J. Mech. Phys. Solids, Vol. 37, pp. 717-733.

Zaslawsky, M., 1973,Multiaxial Stress Studies on Rigid Polyurethane Foam, Exper. Mech., Vol. 13, pp. 70-76. 


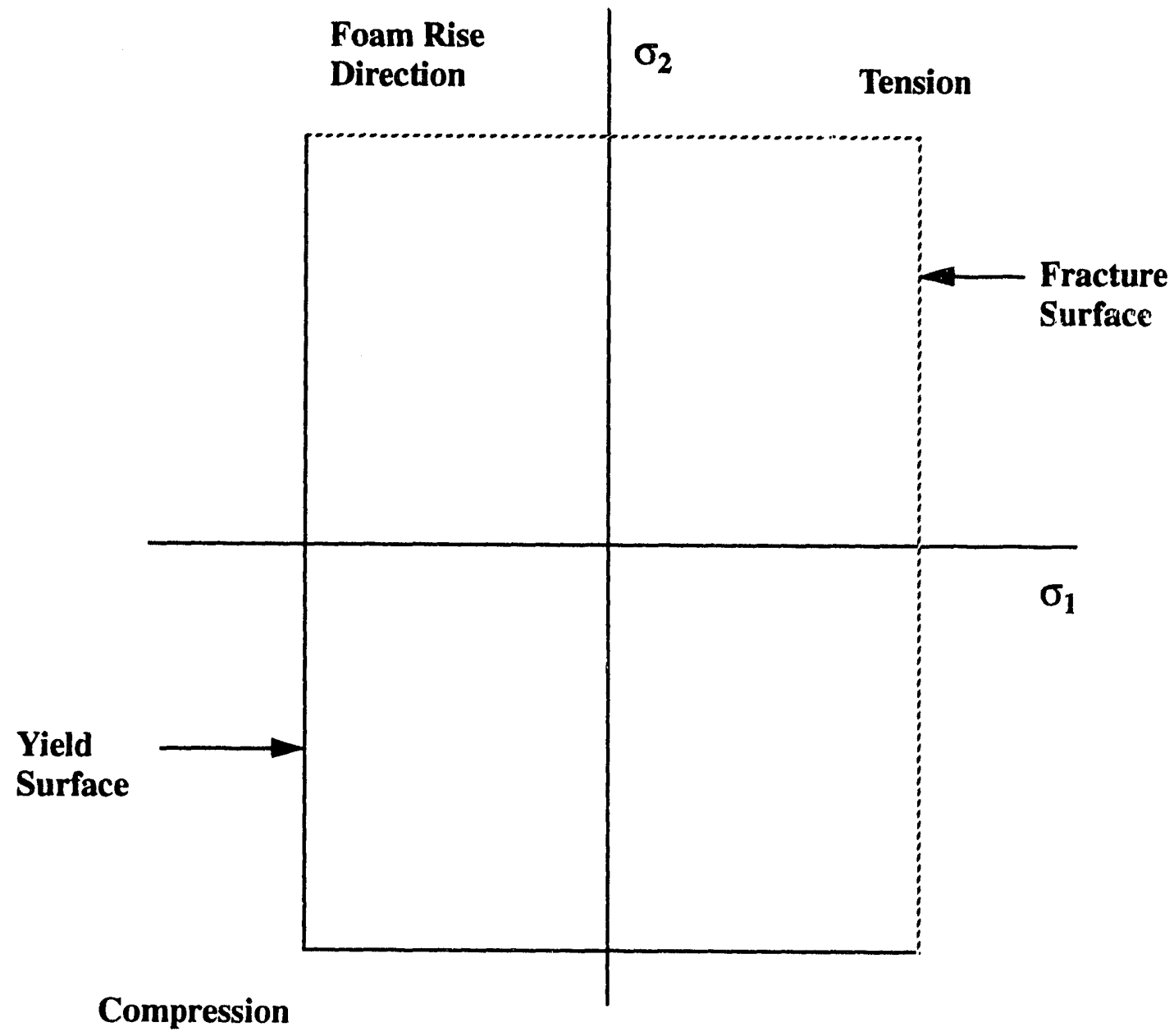

Figure 1. Yield and Fracture Surfaces for a Rigid, Closed-Cell, Polyurethane Foam (Patel and Finnie, 1969; Gibson and Ashby, 1988). 


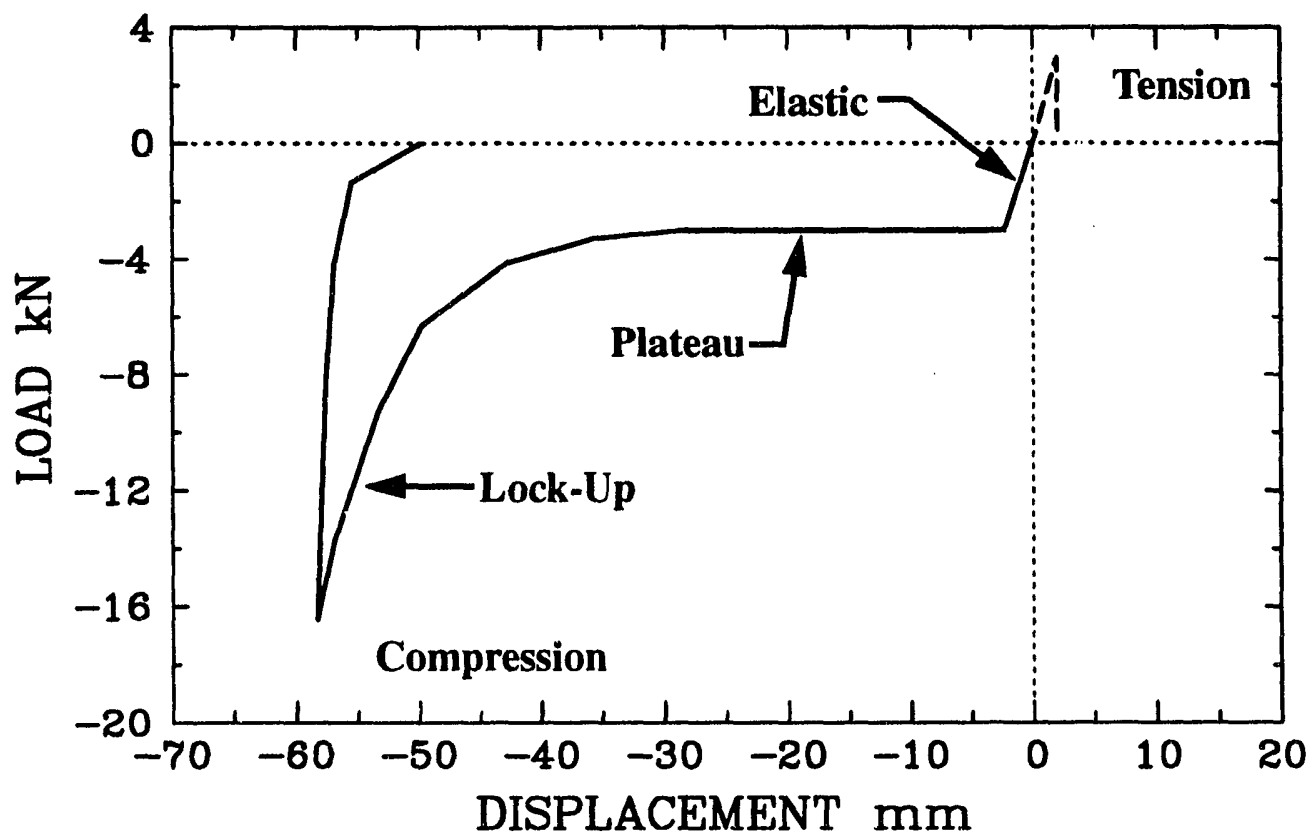

Figure 2. Typical Load-Displacement Curve for $80 \mathrm{~kg} / \mathrm{m}^{3}$ Polyurethane Foam Subjected to Either Uniaxial Compression or Uniaxial Tension (Donald and Maji, 1992).

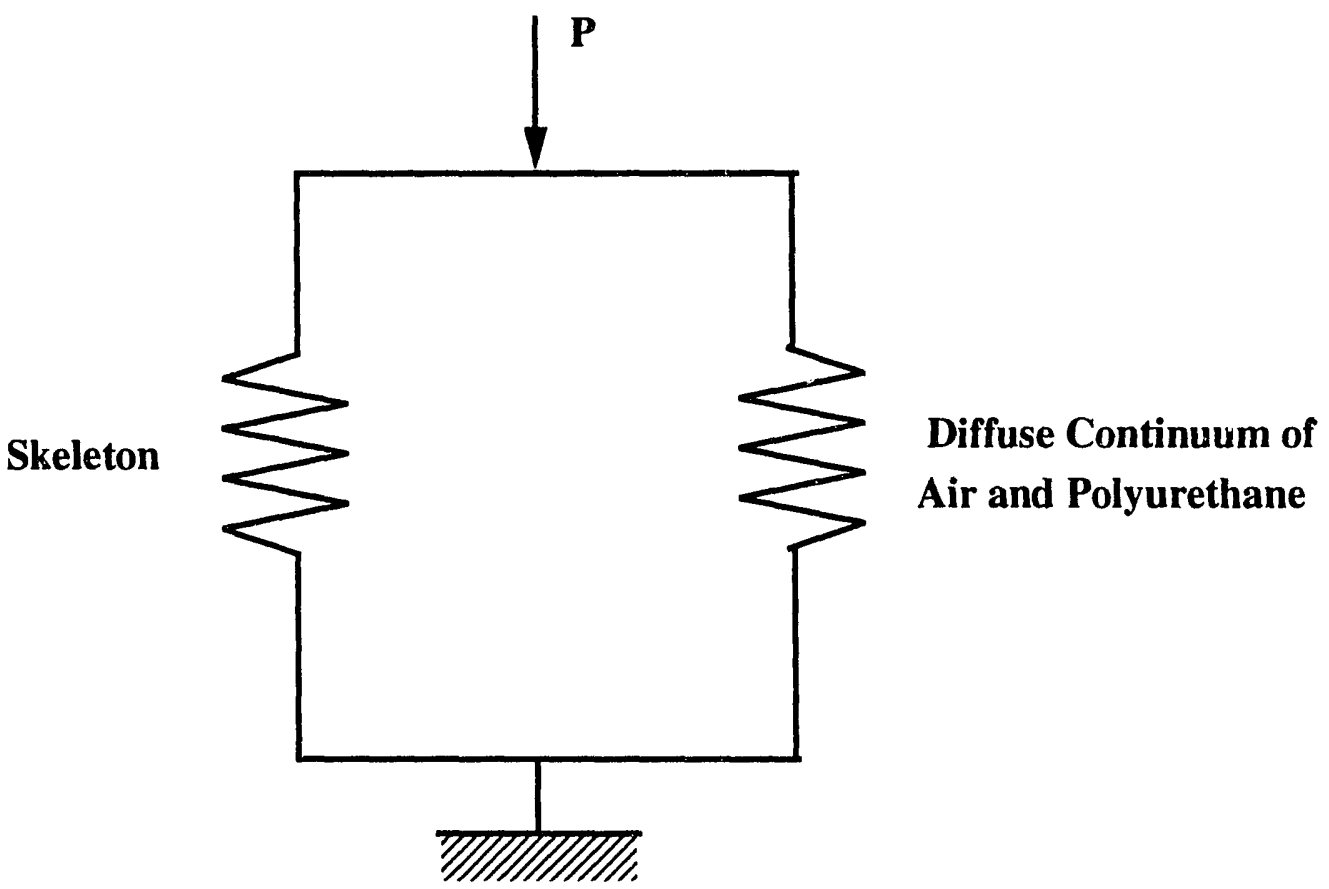

Figure 3. Decomposition of Foam Response into Skeleton and Diffuse Continuum Responses. 


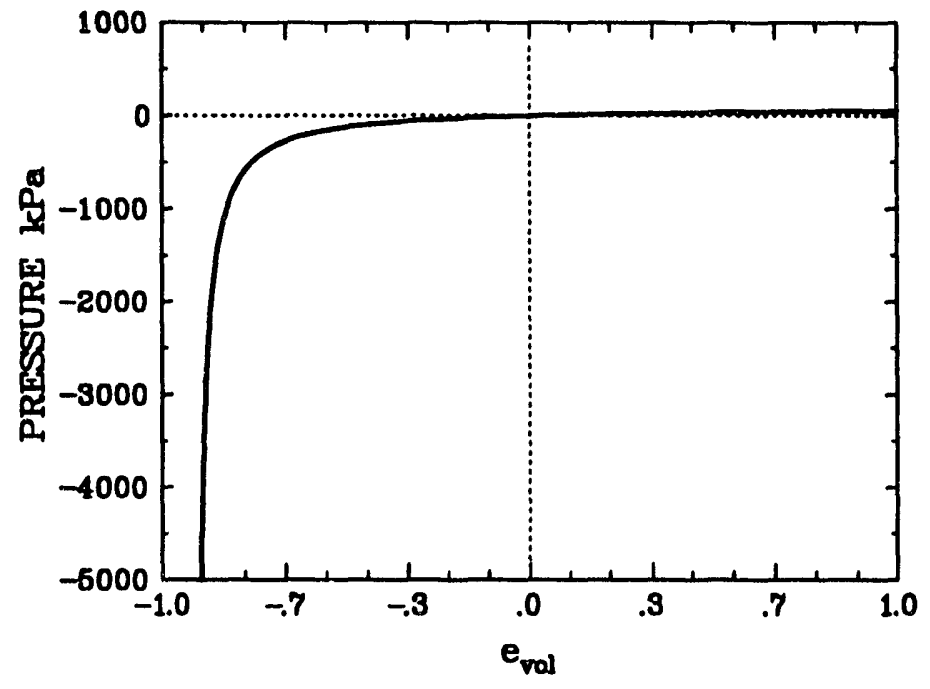

Figure 4. Diffuse Continuum Response for an $80 \mathrm{~kg} / \mathrm{m}^{3}$ Polyurethane Foam.

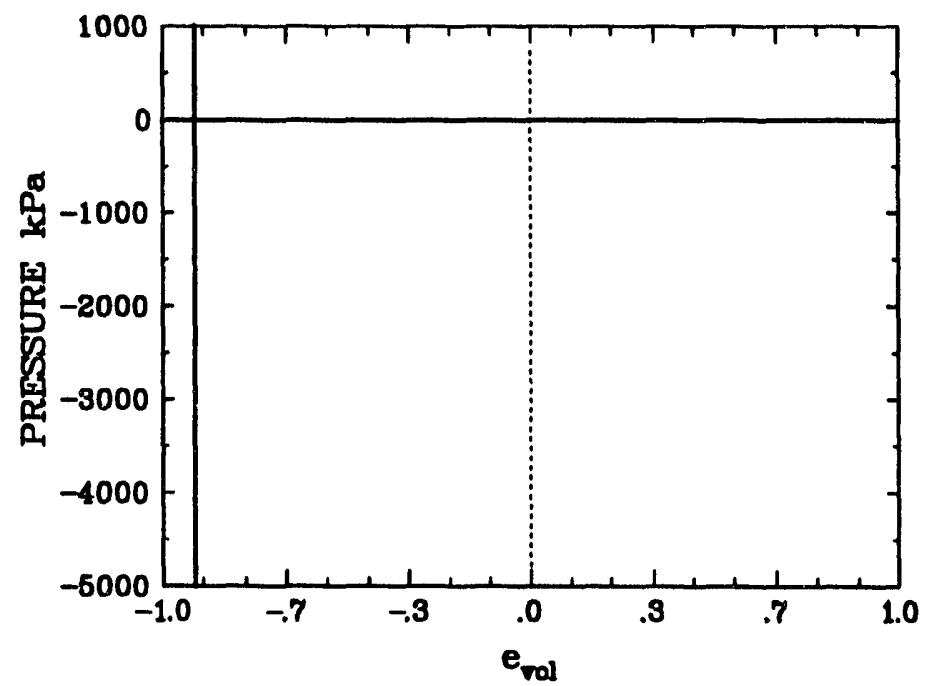

Figure 5. Diffuse Continuum Response for an $80 \mathrm{~kg} / \mathrm{m}^{3}$ Polyurethane Foam. with Air Allowed to Escape. 


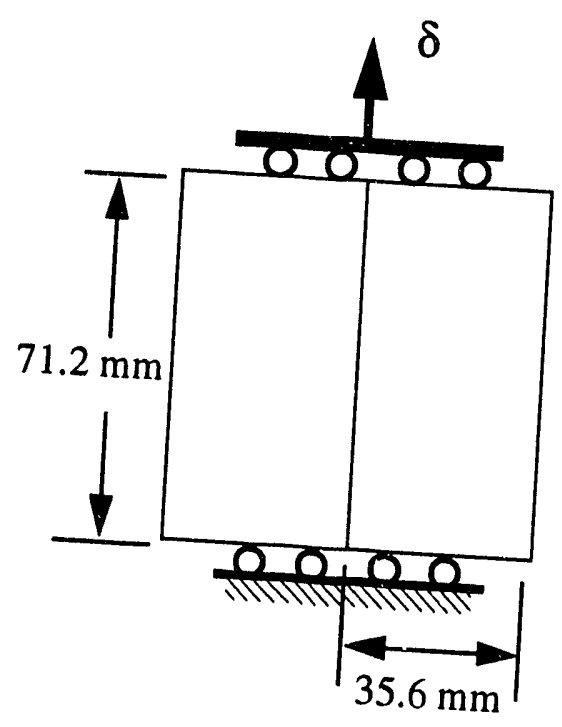

(a) 1 element

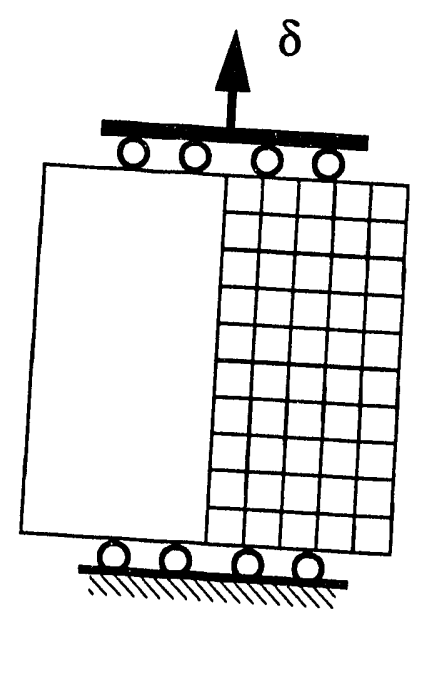

(b) 50 elements

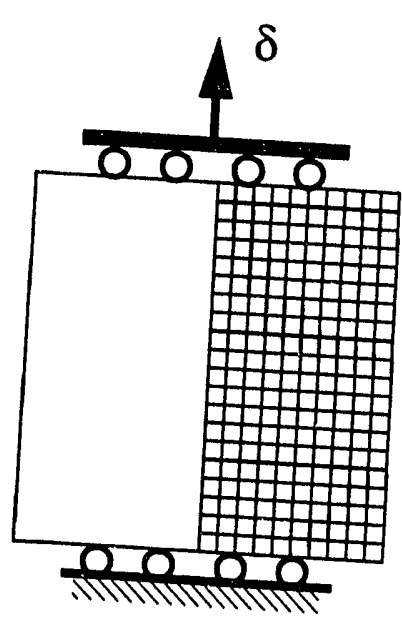

(c) 200 elements

Figure 6. Axisymmetric Finite Element Models of Foam Specimens. 


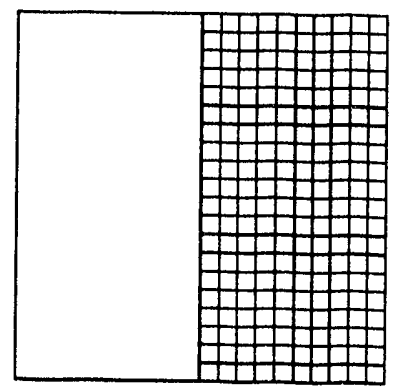

(a) undeformed

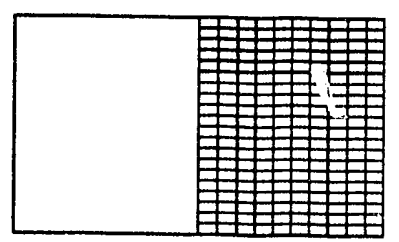

(b) plateau

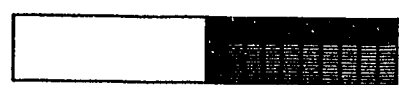

(c) lock-up

Figure 7. Deformed Shape of 200 Element Model Subjected to Uniaxial Compression.

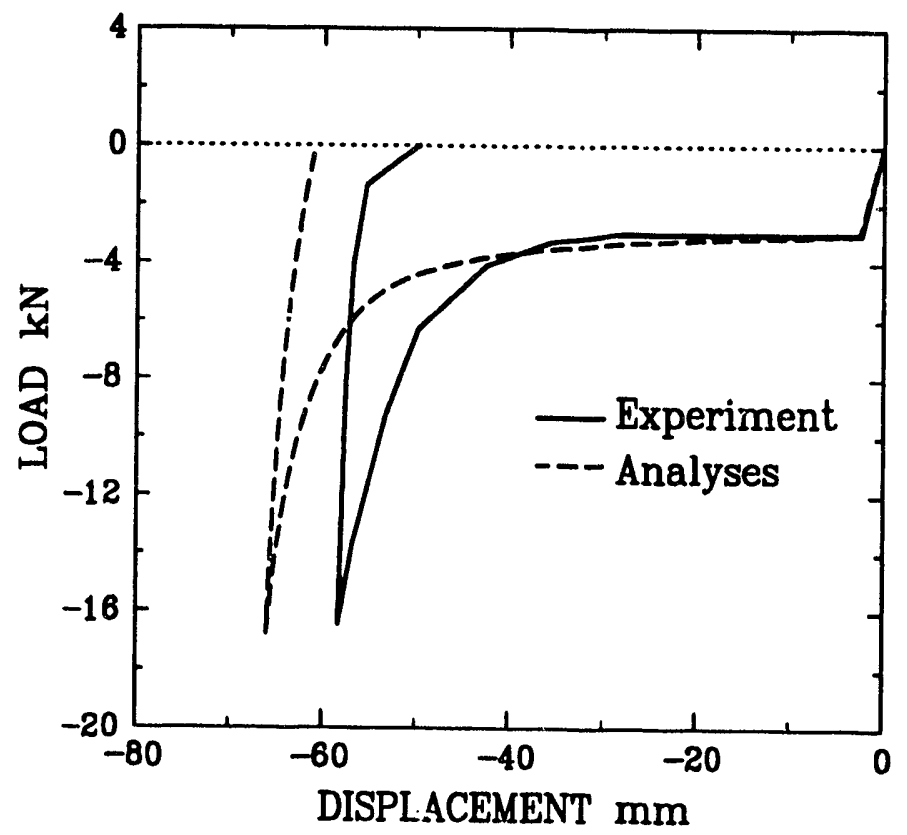

Figure 8. Applied Load vs. End Displacement - Uniaxial Compression -

Comparison of Finite Element Analysis Predictions with Experiments. 


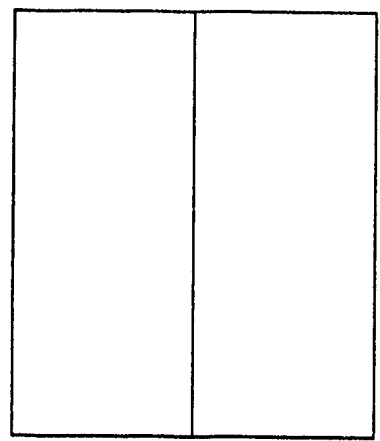

(a) 1 element

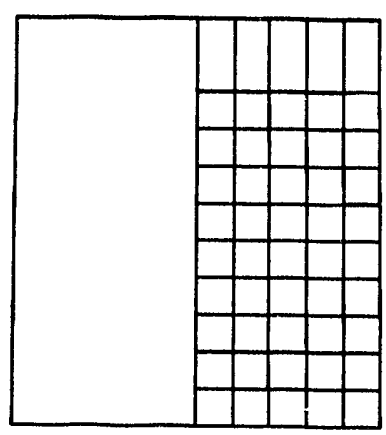

(b) 50 elements

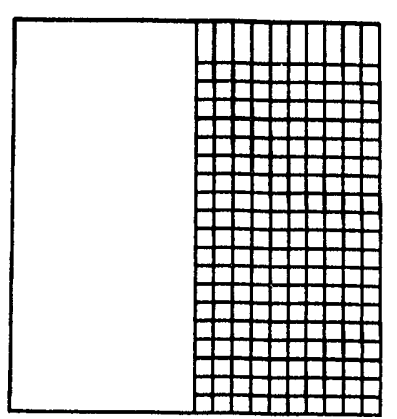

(c) 200 elements

Figure 9. Deformed Shape of Models Subjected to Uniaxial Tension.

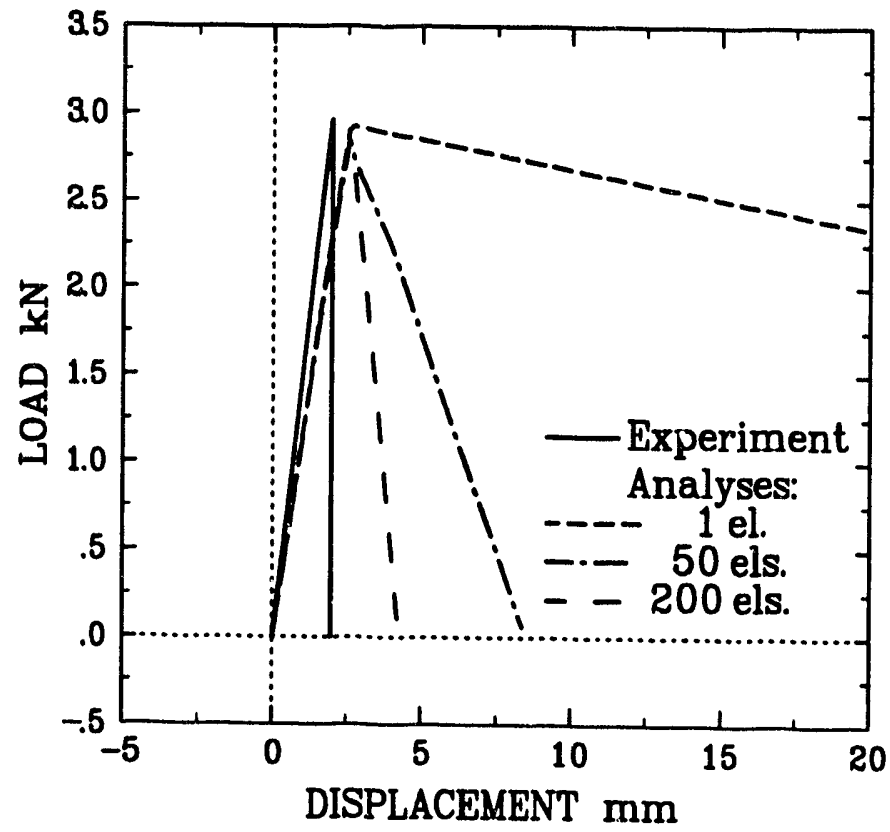

Figure 10. Applied Load vs. End Displacement - Uniaxial Tension -

Comparison of Finite Element Analysis Predictions with Experiments. 


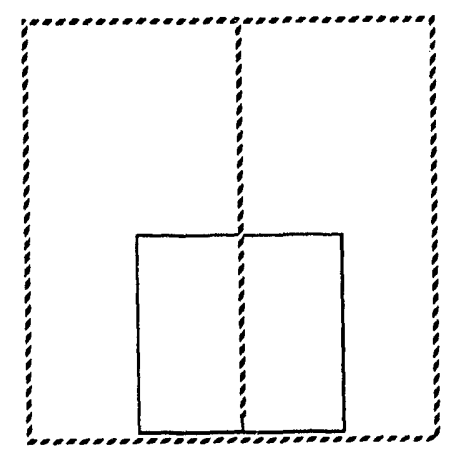

(a) 1 element

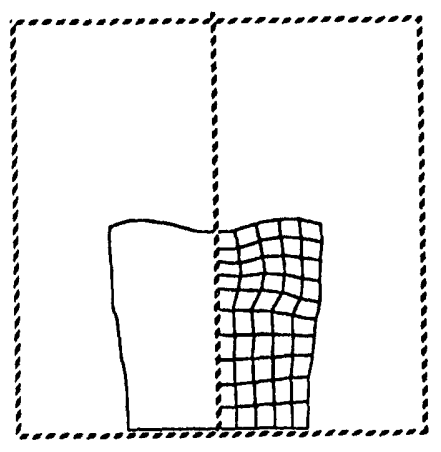

(b) 50 elements

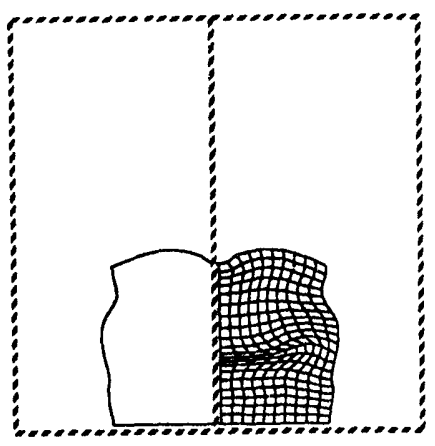

(c) 200 elements

Figure 11. Deformed Shape of Models Subjected to Hydrostatic Compression.

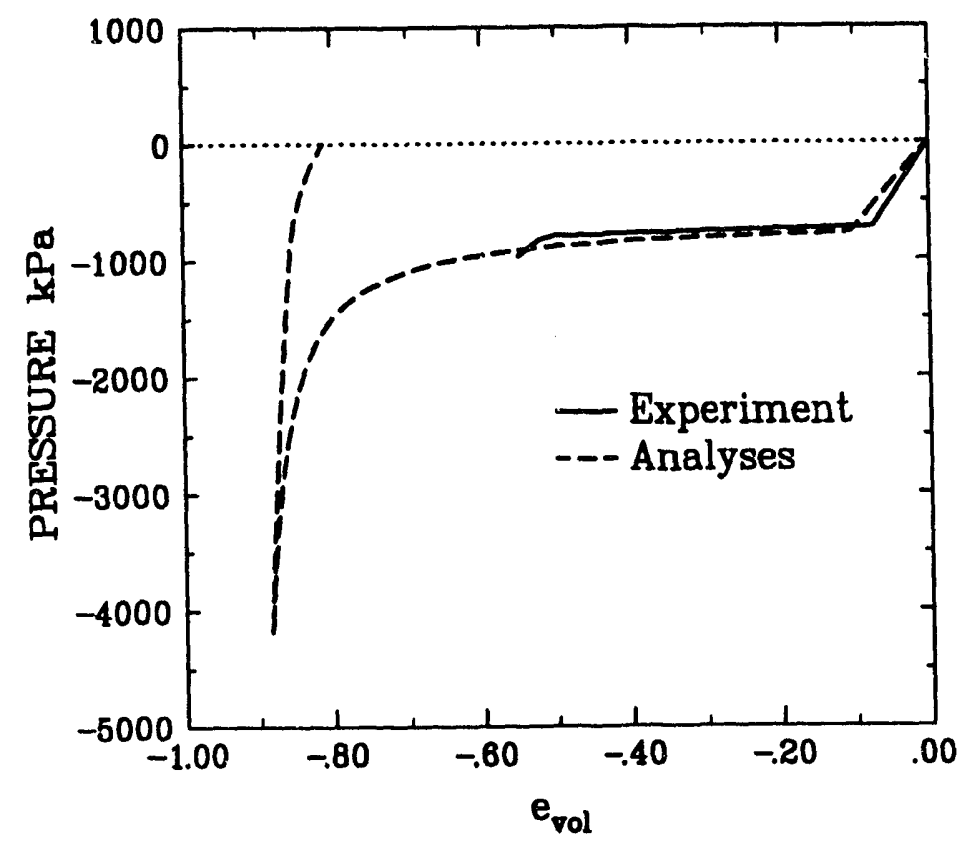

Figure 12. Applied Pressure vs. Engineering Volume Strain - Hydrostatic Compression Comparison of Finite Element Analysis Predictions with Experiment. 

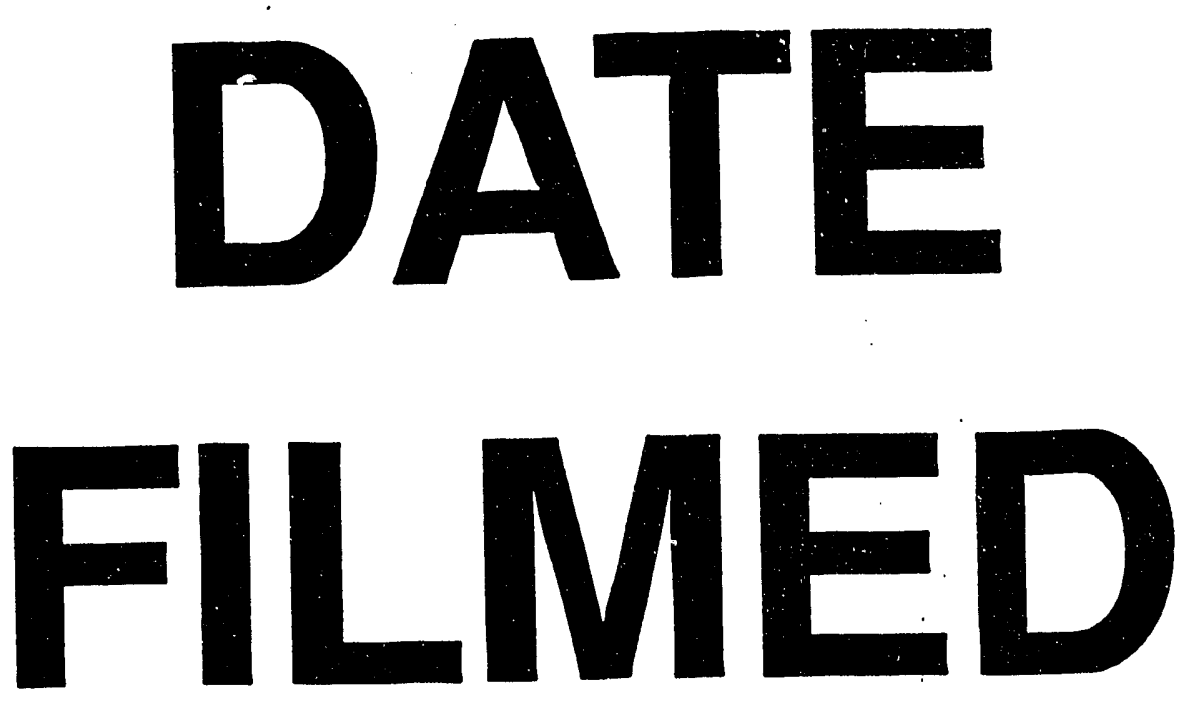

$10 / 13 / 93$
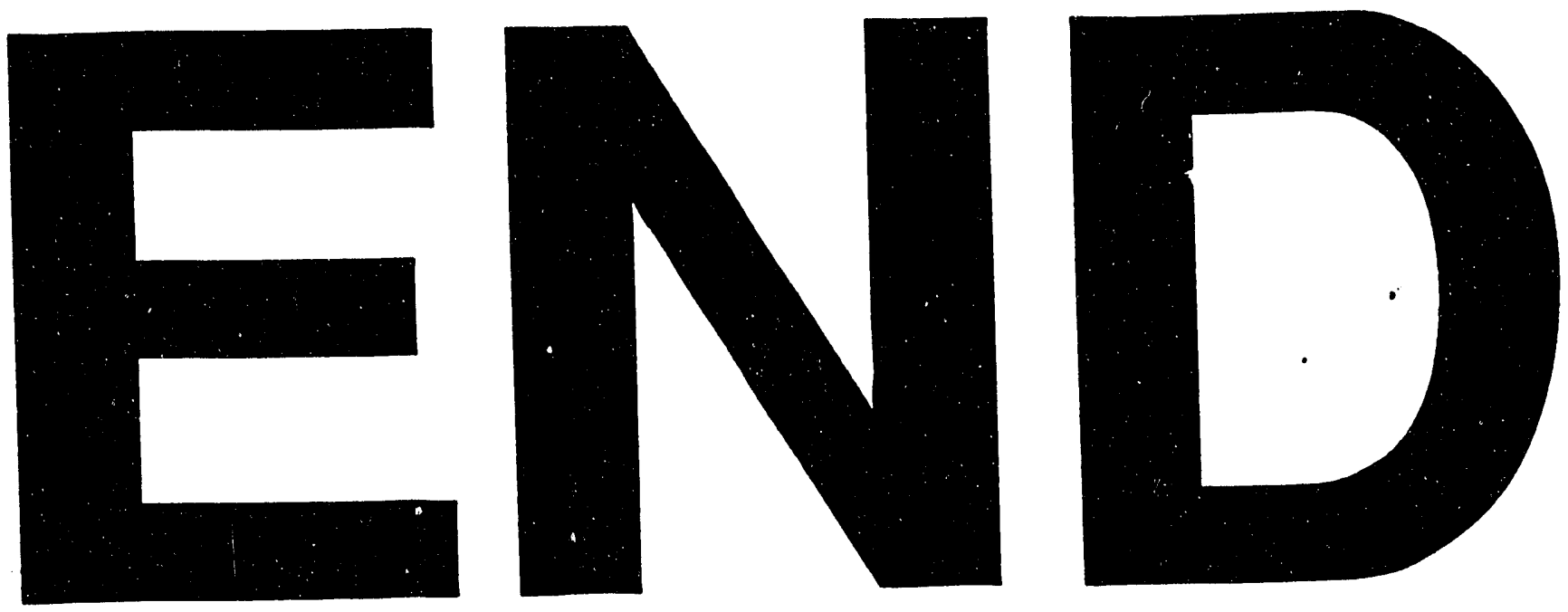
